had increased metabolism, and those with lower D2 measures had decreased metabolism. MPH, like cocaine, binds to the dopamine transporter, but their effects on metabolism are not identical. The cocaine craving induced by MPH in cocaine abusers is not associated with an MPH addiction. In fact, if substance abuse becomes a problem in adolescence, it is independent of ADHD and does not involve addiction to MPH (Biederman J et al, 1997, in Millichap JG. Attention Deficit Hyperactivity and Learning Disorders. PNB Publ, 1998;pp129-130)

The right striato-orbitofrontal regions are thought to be involved in the neuroanatomic and biochemical basis for ADHD. A deficiency in brain dopamine and norepinephrine may explain a loss of inhibition and excessive activity levels in children with ADHD. MPH, by increasing dopamine activity in the right frontal lobe, may normalize motor activity while preserving and heightening the degree of alertness.

\title{
PRENATAL PCB AND DIOXIN AND COGNITIVE DYSFUNCTION
}

The effects of environmental exposure to polychlorinated biphenyls (PCB) and dioxins on cognitive abilities in Dutch children at 42 months of age were assessed at the Sophia Children's Hospital, Rotterdam, The Netherlands. Maternal plasma and breast milk concentrations were compared with scores of cognitive function. Maternal plasma total PCB concentrations were associated with lower cognitive functioning, and the effect was related to the degree of prenatal in utero exposure. Lactational and current childhood exposure to PCBs and dioxins were not related to 42-month cognitive performance. (Patandin S, Lanting CI, Mulder PGH, et al. Effects of environmental exposure to polychlorinated biphenyls and dioxins on cognitive abilities in Dutch children at 42 months of age. L Pediatr Jan 1999;134:33-41). (Reprints: Svati Patandin MD, Department of Pediatrics, Division of Neonatology, SP 3435, Sophia Children's Hospital, PO Box 2060, 3000 CB Rotterdam, The Netherlands).

COMMENT. The developing fetal brain is particularly sensitive to environmental toxins. Prenatal in utero exposure to PCBs causes impaired cognitive performance in children, whereas lactational and childhood exposure may not have adverse effects on learning. These results are in agreement with previous Chinese poisoning and Michigan fish exposure studies. Although deficits are often small, the implications of low-level PCB exposure may be compared to that of lead exposure. Furthermore, the effects on intellectual performance are long-lasting, and ADHD is a potential complication. (Progress in Pediatric Neurology III, PNB Publ, 1997;pp226-8).

\section{ANTIEPILEPTIC DRUGS}

\section{TOPIRAMATE AND OTHER AED EFFECTS ON COGNITION}

The acute and steady-state cognitive effects of three new antiepileptic drugs (AED), gabapentin, lamotrigine, and topiramate, were studied in healthy young adults at the University of Alabama Epilepsy Center, Birmingham, AL. Compared with baseline tests of attention and memory, topiramate (TPM) caused statistically significant declines on measures of attention and word fluency at acute doses, whereas gabapentin (GBP) and lamotrigine (LTG) had minimal effects on performance. Only topiramate subjects had persistent neurocognitive impairments when tested after 2 and 4 weeks of drug administration. The TPM group's verbal fluency rate dropped an average of $50 \%$ per subject, and the visual attention task showed a threefold increase in rate of errors. The adverse effects of 